\title{
Un contexte comparatif international
}

\section{Tjeer Plomp}

Traducteur : Josette Le Coq

\section{CpenEdition}

\section{Journals}

Édition électronique

URL : http://journals.openedition.org/ries/3288

DOI : 10.4000/ries.3288

ISSN : 2261-4265

Éditeur

Centre international d'études pédagogiques

Édition imprimée

Date de publication : 1 septembre 1996

ISSN : 1254-4590

\section{Référence électronique}

Tjeer Plomp, «Un contexte comparatif international », Revue internationale d'éducation de Sèvres [En ligne], 11 | 1996, mis en ligne le 29 juillet 2013, consulté le 01 mai 2019. URL : http:// journals.openedition.org/ries/3288; DOI : 10.4000/ries.3288

Ce document a été généré automatiquement le 1 mai 2019.

(c) Tous droits réservés 


\title{
Un contexte comparatif international
}

\author{
Tjeer Plomp
}

Traduction : Josette Le Coq

\section{NOTE DE L'AUTEUR}

L'auteur remercie William A. Loxley, ancien directeur exécutif de l'IEA, pour son importante contribution aux versions précédentes de cet article

1 Cette contribution présente l'évaluation des élèves dans une perspective comparative internationale. Un aspect important de l'évaluation est sa fonction d'outil de contrôle de la qualité de l'éducation. Fondamentalement, cet article tend à démontrer que l'évaluation est un élément intégral du pilotage des curricula de nos écoles pour atteindre leurs objectifs.

Un autre aspect important est que l'évaluation ne peut être interprétée trop étroitement en ne regardant que les résultats des élèves (via les examens ou les évaluations nationales), donnant ainsi plus d'importance aux acquis des élèves qu'au curriculum enseigné dans les classes et qu'aux objectifs du système dans son entier. À notre avis, le concept de curriculum a plusieurs autres dimensions qui dépassent les données d'évaluation des élèves et les procédures d'évaluation viennent d'autres sources que des élèves. Dans ce chapitre, nous abordons la relation entre curriculum et évaluation en utilisant une définition des multiples facettes du curriculum. De notre point de vue, une perspective comparative internationale n'enrichit pas seulement les pratiques nationales d'évaluation mais aussi ajoute un élément unique à l'interprétation, de même qu'elle éclaire les autres pratiques qui peuvent conduire à l'amélioration du curriculum.

3 Avant de présenter les exemples illustrant comment l'évaluation internationale comparative peut contribuer au pilotage des curricula nationaux, nous donnons les définitions du curriculum et de l'évaluation dans leurs différents contextes. Puisque les exemples sont tirés des recherches de l'IEA (International Association for the Evaluation of 
Educational Achievement), la mission et la portée de l'IEA seront décrites ainsi que la conception et le développement de ses recherches.

\section{Le curriculum vu à trois niveaux}

Ce que les élèves apprennent dépend de ce que les enseignants choisissent de leur enseigner. En amont, ce que les enseignants choisissent d'enseigner dépend des plans de cours, des programmes officiels, des manuels, etc. Ces documents sont préparés et fixés en dehors des établissements sur la base de décisions prises par les autorités nationales (gouvernementales ou régionales) dont se servent, à leur tour, les concepteurs de curriculum, les auteurs et les éditeurs de manuels pour préparer des matériels parmi lesquels les établissements et les enseignants font leur choix. Les curricula peuvent par conséquent être conçus à trois niveaux: la nation, l'établissement et l'élève; chaque niveau peut être mesuré, évalué et comparé pour déterminer le degré de recouvrement et de corrélation. Ceci est présenté dans la figure $1^{1}$.

Figure 1 : Types de curricula utilisés dans les études de l'IEA

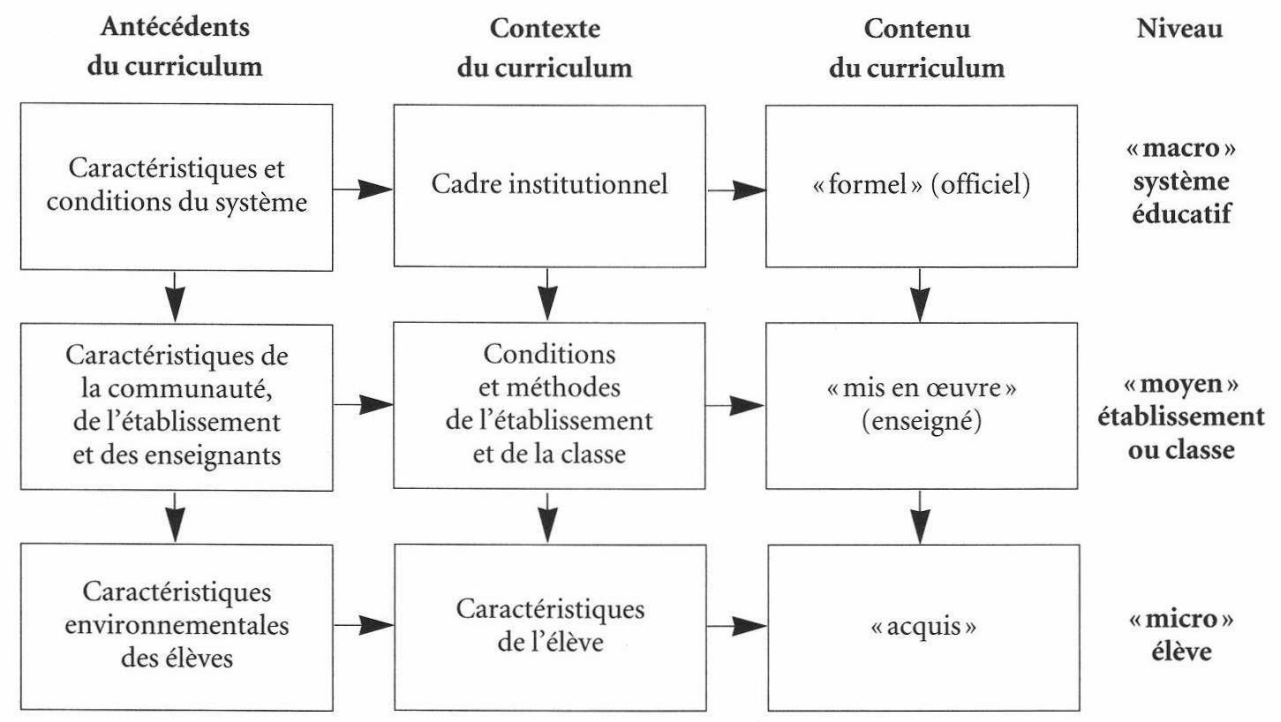

Au niveau « macro » (général) du système éducatif (par exemple celui de la nation ou de la région), il existe le «curriculum formel» (officiel) (intented curriculum) qui est celui stipulé dans les programmes officiels, les plans de cours ou dans les manuels et qui représente les conceptions partagées par les concepteurs, les enseignants et les parents sur ce qui est important et qui pourrait être enseigné et appris à l'école. Au niveau «moyen» (intermédiaire) - celui de l'établissement ou de la classe -, il existe le "curriculum mis en œuvre» (enseigné) (implemented curriculum) qui est formé des contenus de cours, des horaires, des stratégies pédagogiques que l'enseignant utilise réellement dans sa classe. Enfin au niveau « micro » de l'élève, on trouve le « curriculum acquis » (attained curriculum) formé des compétences et des attitudes cognitives de l'élève résultant de l'enseignement et de l'instruction. Ces trois aspects du curriculum sont emboîtés et peuvent être mesurés. Les curricula nationaux peuvent être évalués en examinant les plans nationaux, les manuels, les examens, etc., pour déterminer l'étendue du contenu proposé quelle que soit la classe. Le contenu réel du curriculum des classes 
peut être perçu chez les enseignants et les élèves en utilisant, par exemple, des questionnaires d'enquêtes qui mesurent ce qui est appris, ce qui est enseigné et les représentations (attitudes) que les élèves et les enseignants ont des processus d'enseignement et d'apprentissage. Enfin, les tests et questionnaires peuvent être utilisés pour évaluer ce que les élèves apprennent réellement et quelles sont leurs attitudes vis-àvis d'une discipline.

De plus, les antécédents du curriculum comme le contexte social de la famille de l'élève ou l'environnement de l'établissement aussi bien que ses ressources peuvent être corrélées au contexte du curriculum (par exemple le cadre institutionnel, les processus d'instruction et les comportements des élèves) pour prévoir les résultats des curricula.

7 Les évaluations basées sur cette approche à trois étages permettent l'étude des curricula nationaux à différents niveaux. Qui plus est, quand la même méthodologie et les mêmes instruments sont utilisés dans plusieurs pays, le contexte peut être élargi à un contexte international. Enfin, le modèle général peut être utilisé pour générer et tester une large gamme d'hypothèses à l'intérieur des systèmes éducatifs du monde entier et entre eux, fournissant un moyen puissant d'évaluer la qualité d'un curriculum national en se basant sur des « normes de classe mondiale ».

\section{La portée de l'évaluation}

Nisbet ${ }^{2}$ signale deux utilisations de l'évaluation: la responsabilité et l'évaluation de l'instruction. Le premier emploi se réfère à une responsabilité des autorités éducatives contrôlant l'efficacité du système éducatif dans son ensemble et des établissements à l'intérieur de ce système. La responsabilité est avant tout un outil du niveau "macro ", mais est aussi pertinent pour les administrateurs au niveau "moyen" (le niveau de l'établissement). La deuxième utilisation de l'évaluation fournit une rétroaction sur l'enseignement et l'apprentissage à la fois pour les enseignants et les élèves ${ }^{3 .}$ L'évaluation en éducation est un outil des niveaux « micro » et « moyen » étroitement lié à la mesure des résultats de l'apprentissage basé sur les processus éducatifs. La distinction entre les responsabilités politiques et professionnelles est manifeste dans ces deux utilisations de l'évaluation, et on se demande jusqu'à quel point ces deux emplois de l'évaluation sont compatibles ou conciliables. Examinant des propositions d'évaluation de l'éducation en Norvège, Grandheim et Lundgren ${ }^{4}$ concluent que les deux approches sont vraiment compatibles.

9 La suite de cet article présente des exemples montrant que les études comparatives internationales sur l'évaluation jouent vraiment un rôle important en matière de responsabilité - fournissant de cette façon une rétroaction aux niveaux «macro » et "moyen» des systèmes éducatifs, tout en donnant un aperçu des stratégies et des instruments utilisés pour évaluer l'instruction.

On suppose que l'évaluation est utilisée pour contrôler le contexte aussi bien que le contenu curriculaire. Ce qui signifie que, étant donné les buts et objectifs d'un système éducatif à un niveau quelconque, des données soient recueillies pour juger l'état du système, dans le but de décider ce qu'il est nécessaire de faire pour améliorer l'instruction. En effet, on suppose que les systèmes éducatifs à tous les niveaux sont, en fait, des systèmes d'apprentissage améliorables. Il est important d'inclure les trois niveaux du curriculum car les parents et les élèves ont le droit d'attendre une éducation 
de qualité - installations modernes, curriculum et instruction bien organisés. Contrôler la qualité des curricula formels et mis en œuvre est de la responsabilité des autorités éducatives. Ces autorités ne pratiquent pas le contrôle pour lui-même mais pour améliorer ce que les élèves apprennent (le curriculum acquis), ce que les enseignants enseignent (le curriculum mis en œuvre) et ce que la communauté valorise (le curriculum formel).

11 Étant donné les différents niveaux curriculaires et leurs différents outils d'évaluation, l'évaluation comporte sûrement des approches différentes à chaque niveau. Par exemple, les questions d'une évaluation portent sur :

- le curriculum acquis : ce que les élèves réussissent et ce que sont leurs attitudes, mesurés dans des études internationales à l'aide de tests et de questionnaires sur l'environnement de l'élève, l'exposition au curriculum, etc., administrés à des échantillons aléatoires d'élèves ;

- le curriculum mis en œuvre : ce qui est réellement enseigné dans les établissements appelé « chances d'apprendre » (opportunity to learn, OTL, dans les études IEA), et dans quelles conditions (disponibilité des manuels, enseignants formés, méthodes pédagogiques, égalité des chances pour tous les élèves, installations, etc.);

- le curriculum formel : le contenu et les objectifs du curriculum (à travers les programmes, les normes minimales, les réglementations nationales concernant la qualification des enseignants et le contenu du curriculum, les résultats aux examens, etc.).

\section{L'IEA : mission et méthodes de recherche}

12 L'IEA (International Association for the Evaluation of Educational Achievement), fondée à la fin des années cinquante, est une association indépendante de centres de recherche. Actuellement, environ cinquante-cinq pays sont membres de l'IEA, parmi eux tous les pays membres de l'OCDE. L'IEA a pour mission de conduire des études comparatives sur les politiques et pratiques éducatives à l'intérieur et entre les systèmes éducatifs, dans le but d'améliorer l'apprentissage ${ }^{5}$. Dans ses études ${ }^{6}$. l'IEA ne se concentre pas seulement sur la mesure de la réussite des élèves mais aussi sur les effets des caractéristiques de l'établissement et de la famille sur l'apprentissage.

13 L'IEA reconnaît deux objectifs aux études comparatives internationales de la réussite : fournir aux décideurs politiques et aux praticiens de l'éducation des informations sur la qualité de leur système éducatif en liaison avec des groupes pertinents de référence dans des pays similaires, et aider les chercheurs et les décideurs politiques à comprendre les raisons des différences observées entre les systèmes éducatifs. En menant des études, les chercheurs de l'IEA essaient d'obtenir deux sortes de comparaisons. La première consiste à montrer les influences de l'enseignement sur les scores ou sous-scores internationaux de réussite. La deuxième comparaison cherche à montrer comment le curriculum formel ("ce qui devrait être enseigné») d'un pays donné est mis en œuvre dans les établissements et maîtrisé par les élèves. Une étude caractéristique de l'IEA porte sur des échantillons de classes prises dans trois populations: l'école primaire (primary), le premier cycle secondaire (junior secondary education), le second cycle secondaire (senior secondary education). 


\section{L'impact des recherches de l'IEA au niveau national}

14 comparatives internationales sont utilisés pour aider dans leurs tâches les décideurs politiques et éducateurs nationaux, gouvernementaux (provinciaux) ou régionaux chargés d'améliorer l'efficacité de l'éducation. Les exemples sont tirés des études suivantes : la deuxième enquête internationale sur les sciences, celle sur la lecture et celle sur l'informatique dans l'enseignement.

\section{Les résultats sur les connaissances en sciences}

La seconde étude IEA sur les sciences (SISS) ${ }^{7}$, effectuée au cours des années quatre-vingt, portait sur la réussite en sciences des élèves de 10,14 et 17 ans dans vingt-trois pays au monde. Différents tests furent administrés à des pays sélectionnés ayant les ressources nécessaires pour conduire des tests de performance qui contenaient, en plus des items "papier-crayon» de sciences sous forme de questions à choix multiples, des épreuves pratiques plus approfondies avec trois sous-scores: l'exécution, le raisonnement et l'investigation. La figure 2 donne les scores aux épreuves pratiques et à leurs composantes par rapport aux scores " papier-crayon " pour les élèves de 14 ans dans six pays.

Figure 2 : Réussite aux épreuves pratiques (élèves de 14 ans, 1983-1984 ; d'après Keeves, 1992a)

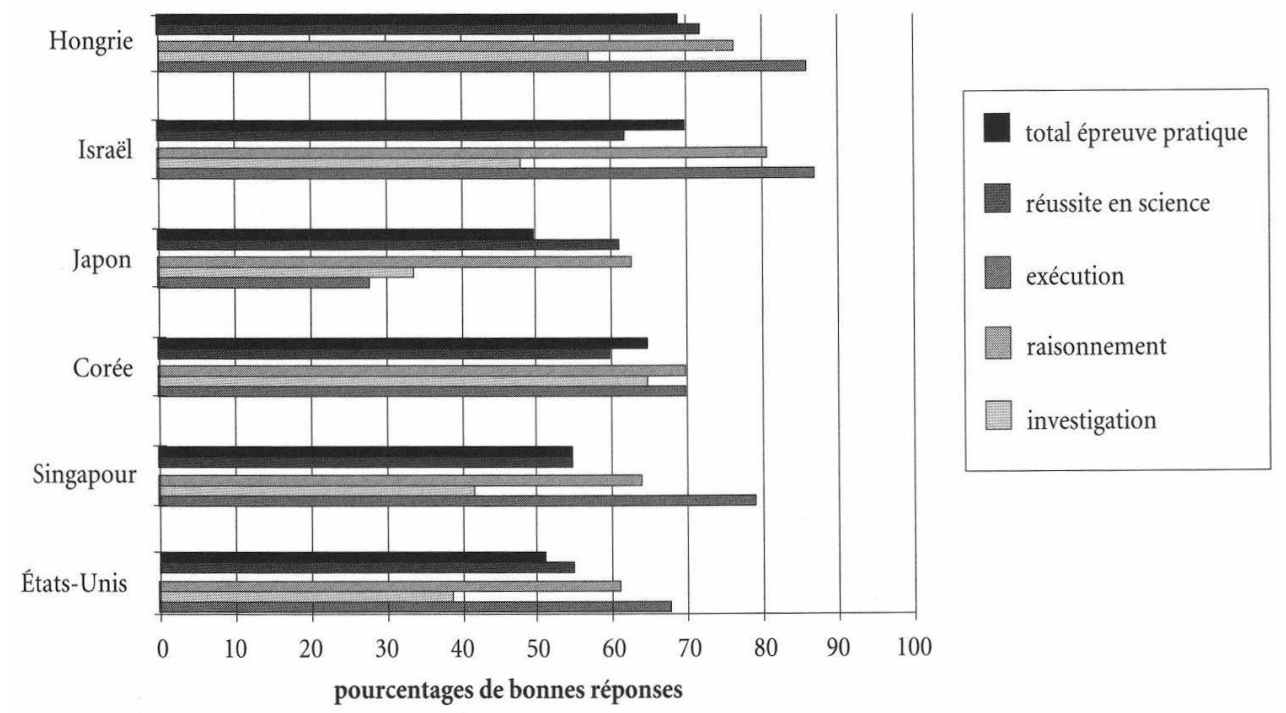

L'analyse du programme des épreuves pratiques menée par Tamir et Doran ${ }^{8}$ montre, par exemple, que les jeunes Japonais réussissent moins bien celles-ci - en particulier en ce qui concerne le raisonnement et l'investigation dans des situations pratiques. Puisqu'il n'y a pas de signe d'une réussite plus faible des élèves japonais aux tests "papier-crayon " portant sur la compréhension ou l'application, les élèves japonais réussissant traditionnellement bien les tests " papier-crayon » de sciences auraient tendance à avoir de faibles performances aux tests qui leur demandent de s'imaginer dans un laboratoire de sciences. Comme le Japon donne peu d'importance à l'enseignement scientifique en 
laboratoire à l'école primaire et au premier cycle secondaire, ce résultat a causé quelque alarme dans les cercles éducatifs japonais9.

17 D'autre part, les élèves américains de 14 ans qui ont des résultats faibles au premier abord, réussissent raisonnablement bien aux épreuves pratiques faisant appel à des méthodes de recherche et d'investigation. Il semble qu'il y ait, aux États-Unis, un lien entre le contenu de la connaissance scientifique et les mesures des compétences de recherche et d'investigation de laboratoire. Lier les tests de performance manuelle aux chances d'apprendre les sciences dans un laboratoire est encore un autre moyen d'examiner le contenu et les méthodes du curriculum mis en œuvre à la lumière des résultats du curriculum scientifique acquis. Les chances d'apprendre en laboratoire influencent nettement le type de processus de pensée scientifique obtenue aux tests écrits et non écrits de sciences.

\section{Les résultats de l'enquête sur la lecture}

18 L'enquête IEA sur la lecture ${ }^{10}$ est encore un autre exemple de recherche internationale fournissant des informations aux décideurs politiques et à la communauté des chercheurs en lecture. Lundberg \& Linnäkylä ont étudié la relation entre les conditions d'enseignement, les caractéristiques des enseignants, les stratégies pédagogiques et les résultats des élèves mesurés par trois sous-scores de lecture : narration, exposition et compréhension de documents. Les buts de l'enseignement de la lecture furent rangés par des enseignantes et classés suivant deux dimensions et quatre pôles ${ }^{11}$.

19 La première dimension est définie par une orientation "technique ", centrée sur l'enseignant (amélioration de la compréhension de textes lus par l'élève, enrichissement du vocabulaire de l'élève, accroissement de la vitesse de lecture), contre une orientation "stratégie ", centrée sur l'élève (développement de la pensée critique de l'élève, extension chez l'élève de son intérêt pour la lecture et de sa vision du monde à travers la littérature).

20 La deuxième dimension s'appuie sur une orientation "littéraire " (la littérature et l'augmentation de l'intérêt pour la lecture, développement d'un intérêt durable pour la lecture, augmentation de l'appréciation de la littérature par l'élève) contre une orientation «fonctionnelle» (accent mis sur l'aspect fonctionnel, développement chez l'élève des méthodes d'études et de recherche, diversification des lectures choisies par l'élève, apprentissage par l'élève de l'application des stratégies d'étude à d'autres matières, apprentissage par l'élève de l'interprétation de diagrammes et graphiques).

21 Les auteurs concluent que l'orientation littéraire est hautement estimée par les enseignants de chaque pays, peut-être parce qu'une grande proportion d'entre eux a été formée d'abord comme professeurs de littérature et ensuite comme experts en lecture. Il semble que les combinaisons de l'orientation fonctionnelle soit avec l'orientation stratégie soit avec l'orientation technique soient rares ou inexistantes, ceci dans au moins trente et un des pays étudiés. Il est remarquable que les combinaisons des orientations technique et littéraire soient représentées principalement par des pays dont les résultats sont faibles. Ces données peuvent fournir aux décideurs politiques en éducation et aux experts en lecture l'occasion de se demander si, étant donné les résultats actuels de plus de trente pays au monde, il est encore admissible que si peu d'attention soit portée à l'orientation fonctionnelle de l'enseignement de la lecture. 

ou intégrés dans une autre discipline - mène-t-elle à l'enseignement de l'informatique et influence-t-elle les connaissances des élèves en informatique? L'enquête IEA sur les ordinateurs dans l'enseignement (COMPED) ${ }^{12}$ examine la diffusion des ordinateurs dans les établissements de vingt et un pays au monde en 1989. Un suivi a eu lieu en 1992 et les indications de tendance sont par conséquent aussi disponibles ${ }^{13}$. Cette étude a révélé un phénomène intéressant en ce qui concerne la diffusion et la mise en œuvre de l'utilisation des ordinateurs à l'école - une découverte utile pour le gouvernement néerlandais. Un des objectifs de la politique d'incitation du gouvernement néerlandais dans les années quatre-vingt a été d'encourager l'intégration de l'emploi d'ordinateurs dans les disciplines scolaires existantes. Dans le cadre de l'étude internationale comparative, des données furent recueillies concernant l'emploi d'ordinateurs en mathématiques, en langue maternelle et en sciences aussi bien qu'à propos de l'initiation aux ordinateurs. La figure $3^{14}$ révèle qu'en 1989, les ordinateurs n'étaient pas beaucoup utilisés dans ces trois disciplines au premier cycle secondaire. Cependant, en 1992, l'emploi d'ordinateurs avait doublé.

Figure 3 : Pourcentage de professeurs de premier cycle secondaire dans des établissements utilisant des ordinateurs à des fins pédagogiques; en 1989 et 1992 (d'après Brummelhuis \& Plomp, 1992)

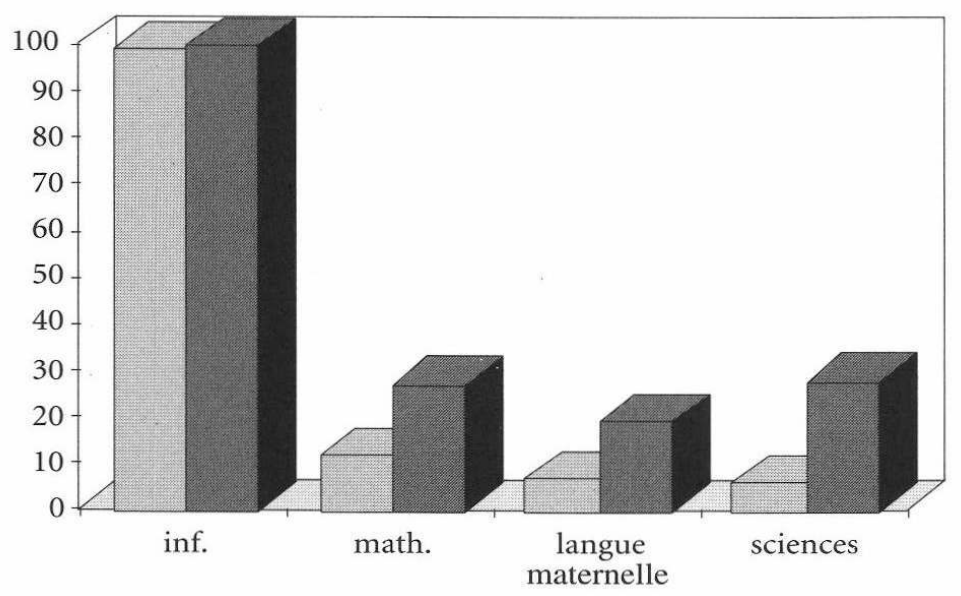
et initiation aux ordinateurs

Les données de COMPED montrent que l'accroissement de l'utilisation des ordinateurs dans ces trois disciplines est presque complètement attribué aux enseignants travaillant 
dans un « département disciplinaire » où en 1989 aucun ordinateur n'était utilisé par les professeurs (cf. figure 3). On peut déduire de ces données que l'objectif politique d'élargir l'emploi des ordinateurs dans les disciplines existantes a été atteint à ce niveau de l'établissement. Mais, comme le montre la figure 4, au niveau des "départements disciplinaires » à l'intérieur des établissements, il y a une petite augmentation du nombre des professeurs utilisant les ordinateurs pour enseigner. Ces données IEA révélèrent aux autorités néerlandaises un fait simple: l'utilisation d'ordinateurs dans les disciplines existantes dans les classes du premier cycle secondaire n'est pas ancrée dans le curriculum mais est plutôt une activité qui dépend du professeur. Ce type d'information peut conduire à des mesures spéciales destinées à stimuler l'intégration des ordinateurs dans le curriculum scolaire et à modifier de façon importante la programmation du curriculum de plusieurs matières pour renforcer l'utilisation des ordinateurs.

Figure 4 : Utilisation de l'ordinateur dans des disciplines par des enseignants qui ont commencé à s'en servir entre 1989 et 1992 (d'après Brummelhuis \& Plomp, 1992)

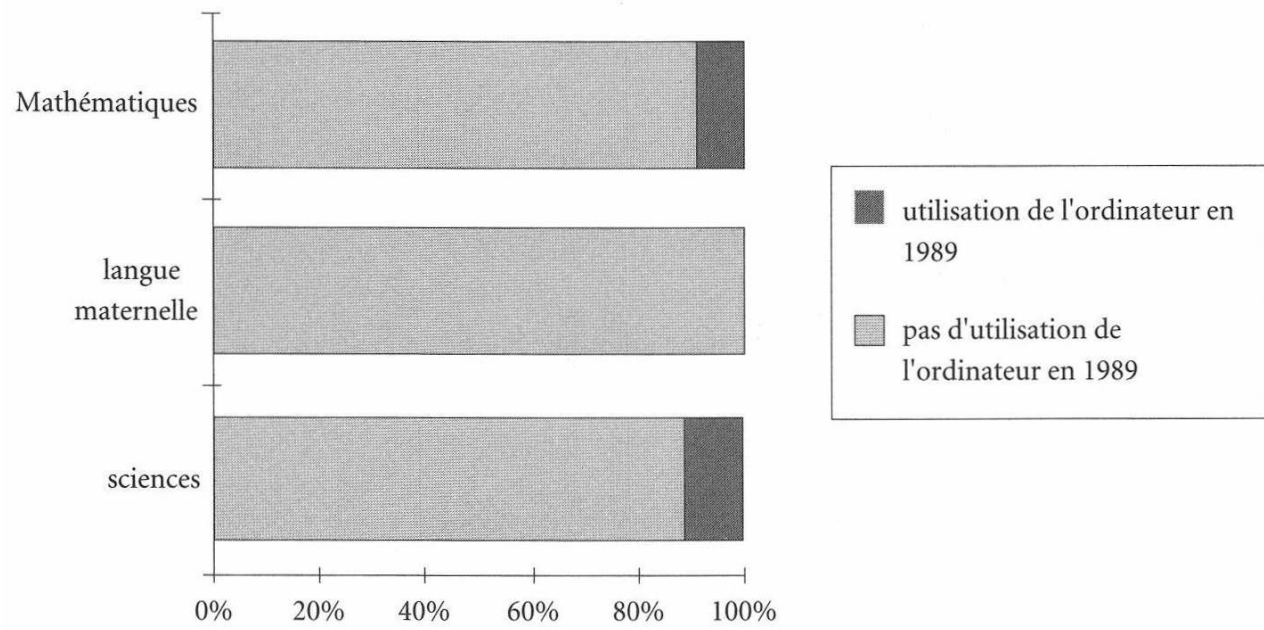

Les gouvernements emploient de nombreux outils pour contrôler la qualité de l'éducation et par conséquent font attention aux réformes du curriculum et aux innovations. Les examens et les évaluations nationales recueillent des données sur la réussite des élèves mais sont seulement deux des nombreux outils d'évaluation disponibles pour les décideurs politiques.

Cet article s'est concentré sur deux aspects de la relation entre curriculum et évaluation.

D'abord on peut soutenir que l'évaluation dans un contexte curriculaire doit comporter davantage que la seule évaluation de la réussite des élèves. En introduisant trois aspects du curriculum - le curriculum formel, celui mis en œuvre et l'acquis, on peut montrer que le curriculum est présent aux trois niveaux du système éducatif, niveau national, niveau de la classe et niveau individuel, et par conséquent il peut être évalué à chacun de ces niveaux. On peut ensuite indiquer qu'en étudiant le curriculum national dans un contexte international des occasions uniques se présentent d'étudier les multiples questions associées à la qualité d'un programme curriculaire. En utilisant les exemples des études passées de l'IEA, ces points furent confirmés. Les études comparatives internationales fournissent l'occasion d'examiner un système curriculaire in situ par comparaison avec d'autres systèmes. De plus, les enquêtes internationales offrent aussi la possibilité d'options nationales fournissant aux autorités éducatives des occasions uniques d'analyser plus en détail ces modèles (patterns) et organisations de leur propre système 
éducatif. A l'occasion de la troisième enquête internationale sur les mathématiques et les sciences de l'IEA, à laquelle participent quarante pays, plusieurs approches nouvelles ont été utilisées pour évaluer la réussite des élèves en mathématiques et en sciences en tenant compte de l'environnement du curriculum qui est enseigné dans la classe et des objectifs du système dans son entier. Les premiers résultats de cette étude seront publiés en novembre 1996.

\section{NOTES}

1. R. A. Garden, «The Second IEA Mathematics Study », Comparative Education Review, 1987, 31 (1), p. 47-68.

K. J. Travers \& I. Westbury (Eds.), The IEA Study of Mathematics I: International Analysis of Mathematics Curricula, Oxford, UK, Pergamon Press, 1989.

2. J. Nisbet, Curriculum Reform: Assessment In Question, Paris, France, OCDE, 1993, p. 29.

3. Nisbet, op. cit. p. 30.

4. M. Granheim \& Lundgren, «Steering by Goals and Evaluation in the Norwegian Education System. A Report from the EMIL Project », Journal Curriculum Studies, 1991, 31 (6), p. 481-505.

5. W. A. Hayes (Ed.), IEA Guidebook: Activities, Institutions and People, Amsterdam, IEA, 1993.

6. Au fil des années, l'IEA a mené plusieurs études sur différentes disciplines scolaires telles que les mathématiques $(1966,1981)$, les sciences $(1974,1983)$, l'anglais et le français comme langues étrangères (1973/74), l'éducation civique (1973/1974), l'écriture (1984/1985), la lecture (1991). En plus des enquêtes sur les disciplines, l'IEA a étudié l'environnement de la classe (1982), l'éducation préscolaire $(1988,1992)$, et l'informatique dans l'enseignement $(1989,1992)$. Une importante étude sur deux disciplines, la «Troisième enquête internationale sur les mathématiques et les sciences" (TIMSS : Third International Mathematics and Science Study) est en voie d'achèvement, les données ont été recueillies en 1994 et 1995 et le premier rapport international sera publié fin 1996. D’autres études sont prévues : étude sur l'enseignement des langues et étude sur l'éducation civique.

7. J.-P. Keeves, Learning Science in a Changing World: Cross-national Studies of Science Achievement: 1970 to 1984, Amsterdam, IEA, 1992a.

J.-P. Keeves (Ed.), The IEA Study of Science III: Changes in Science Education and Achievement: 1970 to 1984, Oxford, Pergamon Press, 1992b.

T. N. Postlethwaite \& D. Wiley (eds.), The IEA Study of Science II: Science Achievement in Twenty-three Countries, Oxford, Pergamon Press, 1992. P. Tamir \& R. Doran (eds.), "Practical Skills Testing » Studies in Educational Evaluation, 18 (3), Oxford, Pergamo Press, 1992.

8. P. Tamir \& R. Doran (Eds.), op. cit.

9. R. Watanabe, «How Japan makes use of international education survey research » in: Loxley, W. (ed.), « Monitoring the Quality of Education Worldwide: II. A Few National Examples of lEA's Impact », Prospects, Quarterly Review of Education, vol. XXII, n 4 (84), p. 455-462, Paris, UNESCO, 1992.

10. W. Elley, How in the World do Students Read?, Amsterdam, IEA, 1992.

W. Elley (Ed.), The IEA Study of Reading Literacy: Achievement and Instruction in Thirty-two School Systems, Oxford, Pergamon Press, 1994.

T. N. Postlethwaite \& K. Ross, Effective Schools in Reading: Implications for Educational Planners, 
Amsterdam, IEA, 1992.

I. Lundberg \& P. Linnäkylä, Teaching Reading Around the World, Amsterdam, IEA, 1993.

H. Wagemaker et al., Gender Differences in Reading Literacy in 32 Countries, Amsterdam, IEA, 1996.

11. I. Lundberg \& P. Linnäkylä, op. cit.

12. W. J. Pelgrum \& Tj. Plomp, The Use of Computers in Education Worldwide: Results from the IEA 'Computers in Education' Survey in 19 Education Systems, Oxford, Pergamon Press, 1991.

W. J. Pelgrum \& Tj. Plomp, The IEA Study of Computers In Education I: Implementation of an Innovation in Twenty Countries, Oxford, Pergamon Press, 1993.

13. W. J. Pelgrum, I.A.M. Janssen Reinen \& Tj. Plomp, (eds.), Schools, Teachers, Students and Computers: a Cross-national Perspective (IEA COMPED Study Stage 2), Amsterdam, IEA, 1993.

14. A. Ten Brummelhuis \& Tj. Plomp, Resultaten van onderzoek naar computergebruik in het onderwijs (Results of research on educational computer use), The Hague, The Netherlands, Ministry of Education and Sciences, Opstap, 44, 1992.

\section{RÉSUMÉS}

Après une analyse de la notion de curriculum à trois niveaux, l'auteur montre, à partir d'exemples, comment les enquêtes réalisées par l'IEA, non seulement enrichissent les informations de chaque pays par les comparaisons qu'elles permettent, mais aussi contribuent au pilotage des curricula nationaux.

\section{INDEX}

Mots-clés : analyse comparative, programme d'enseignement, recherche comparative, curriculum, IEA

\section{AUTEURS}

\section{TJEER PLOMP}

Président de l'IEA, université de Twente, Enschede, Pays-Bas 